\title{
Prevalência e fatores associados ao uso de álcool e tabaco em universitários do curso de enfermagem
}

\author{
Luciana Zaranza Monteiro ${ }^{1}$, Andrea Ramírez Varela², Leonardo Rodrigues Alves ${ }^{3}$, Maria Raimunda Silva Santos ${ }^{4}$, \\ Guilherme Ramos Lopes ${ }^{5}$, Marco Antônio Caetano Júnior ${ }^{6}$, Suderlan Sabino Leandro ${ }^{7}$
}

\section{RESUMO}

Objetivou-se estimar a prevalência e fatores associados ao consumo de álcool e tabaco entre estudantes do curso de Enfermagem. Estudo transversal com 974 acadêmicos, utilizando questionário sobre dados socioeconômicos, uso de álcool, tabaco e percepção de saúde, realizando análises descritivas e regressão logística. Dos participantes, $78,8 \%$ eram mulheres e $62,6 \%$ tinham idade entre 20 a 29 anos. Sobre o consumo de álcool, 65,1\% fazem uso; a prevalência de estudantes classificados em risco para o alcoolismo foi de 3,0\%. Em relação hábito de fumar antes de ingressar na universidade, $81,8 \%$ das mulheres e $68,8 \%$ dos homens adquiriram esse hábito $(p<0,05)$. Os estudantes que moravam com os pais ou parentes apresentaram maior prevalência de tabagismo $(22,8 \%)$ e de álcool $(66,3 \%)$ e, quanto mais baixa a autopercepção de saúde, maior o consumo. Os resultados destacam prioridades nas ações preventivas a população adolescente, pois muitos adquiriram os hábitos antes de ingressar no ensino superior.

Descritores: Etanol; Tabaco; Prevalência; /prevenção \& controle; Estudantes de Enfermagem.

\footnotetext{
${ }^{1}$ Educadora Física e Fisioterapeuta, Doutora em Ciências Médicas. Professora Assistente do Centro Universitário do Distrito Federal. Brasília, DF, Brasil. E-mail: lucianazaranza@hotmail.com.

2 Médica, Mestre em Saúde Pública. Discente do Programa de Pós-Graduação em Epidemiologia, nível Doutorado, da Universidade Federal de Pelotas. Pelotas, RS, Brasil. E-mail: aravamd@gmail.com.

${ }^{3}$ Discente do curso de Graduação em Enfermagem do Centro Universitário do Distrito Federal. Brasília, DF, Brasil. E-mail: leonardoalves707@hotmail.com.

${ }^{4}$ Discente do curso de Graduação em Enfermagem do Centro Universitário do Distrito Federal. Brasília, DF, Brasil. E-mail: rayhijadedios@gmail.com. ${ }^{5}$ Educador Físico, Mestre em Educação Física. Discente do Programa de Pós-Graduação em Educação Física, nível Doutorado, da Universidade de Brasília. Professor Substituto da Universidade de Brasília. Brasília, DF, Brasil. E-mail: guilhermelopes@hotmail.com.

${ }^{6}$ Educador Físico, Mestre em Educação Física. Discente do Programa de Pós-Graduação Stricto sensu em Educação Física, nível Doutorado, da Universidade Católica de Brasília. Brasília, DF, Brasil. E-mail: E-mail: marcohand85@gmail.com.

${ }^{7}$ Enfermeiro, Doutor em Enfermagem. Coordenador do curso de Graduação de Enfermagem do Centro Universitário do Distrito Federal. Brasília, DF, Brasil. E-mail: suderlan.leandro@gmail.com.
}

Artigo recebido: 07/02/2017.

Artigo aprovado: 09/05/2018.

Artigo publicado: 31/12/2018.

\section{Como citar esse artigo:}

Monteiro LZ, Varela AR, Alves LR, Santos MRS, Lopes GR, Caetano Júnior MA et al. Prevalência e fatores associados ao uso de álcool e tabaco em universitários do curso de enfermagem. Rev. Eletr. Enf. [Internet]. 2018 [acesso em: ];20:v20a44. Disponível em: https://doi.org/10.5216/ree.v20.45296. 


\section{INTRODUÇÃO}

A prevalência do uso de drogas no mundo permanece estável, segundo o Relatório Mundial sobre Drogas de 2016, do Escritório das Nações Unidas sobre Drogas e Crime (UNODC) ${ }^{(1)}$, estima-se que um total de 246 milhões de pessoas, ou 5\% da população global entre 15 e 64 anos de idade, usaram drogas ilícitas em 2013. Usuários de drogas problemáticos, por outro lado, somaram por volta de 27 milhões, cerca de 0,6\% da população adulta mundial, ou uma em cada 200 pessoas $^{(1)}$.

Mais da metade da população das Américas e da Europa já experimentou álcool alguma vez na vida(2). 0 consumo de drogas ilícitas atinge 4,2\% da população mundial, sendo a maconha a mais consumida (144 milhões de pessoas), seguida pelas anfetaminas (29 milhões), cocaína (14 milhões) e os opiláceos (13,5 milhões) $)^{(3)}$.

$\mathrm{Na}$ atualidade, no Brasil e no mundo, o uso e o abuso de álcool e/ou outras drogas lícitas têm crescido de forma progressiva entre as pessoas de todas as classes sociais, especialmente entre os jovens ${ }^{(4)}$. Constitui um problema de saúde pública que tem merecido maior atenção na atualidade devido aos seus determinantes e condicionantes culturais, ético-legais, políticos, econômicos e tecnológicos gerando impacto sobre o indivíduo, a família e a sociedade ${ }^{(5)}$. Assim, requer atenção não somente dos pais e responsáveis pelo jovem, mas também da sociedade, dos educadores, profissionais de saúde e da comunidade científica(6).

Atualmente cerca de 3,3 milhões de mortes anuais (corresponde a $6 \%$ de todas as mortes no mundo) estão direta ou indiretamente relacionadas ao consumo de álcool ${ }^{(7)}$. Indivíduos jovens (entre 20 e 49 anos) são as principais vítimas em relação às mortes associadas ao uso do álcool, representando uma importante perda de pessoas economicamente ativas ${ }^{(7)}$.

O consumo de tabaco é responsável por cerca de seis milhões de mortes por ano no mundo ${ }^{(7)}$. Em 2011, o tabagismo foi fator causal de 14.072 óbitos no Brasil, o que corresponde a 14,7\% do total de óbitos ocorridos no país. Hoje em dia, o tabaco mata mais do que o alcoolismo, AIDS, acidentes de trânsito, homicídios e suicídios juntos, o que representa uma situação preocupante para autoridades mundiais ${ }^{(8)}$.

No Brasil, a prevalência de uso de álcool na vida entre universitários das capitais de estados brasileiros no ano de 2009 foi de $86 \%$ e $22 \%$ faziam consumo com risco para desenvolvimento de dependência ${ }^{(9)}$. Isto é preocupante pois o uso desta substância tem sido relacionado ao menor desempenho acadêmico, a problemas orgânicos, sociais e comportamentais, ao uso de outras drogas e também pode levar ao prejuízo no exercício profissional(10).

O ingresso no ensino superior proporciona aos jovens uma alteração no estilo de vida, principalmente quando estes se encontram longe de casa, o que ocorre na maioria das vezes, levando-os a adotarem hábitos errôneos em relação à saúde, os quais poderão perpetuar durante toda a vida, trazendo-lhes prejuízos para o presente e para o futuro(11).

O uso e o consumo excessivo de álcool e outras substâncias psicoativas podem reduzir a expectativa de vida do estudante universitário, uma vez que o predispõe a acidentes automobilísticos, episódios de violência interpessoal, relações sexuais desprotegidas e transmissão do HIV, distúrbios do sono, mudanças do hábito alimentar, redução da percepção e estresse, além de causar prejuízos acadêmicos ${ }^{(12)}$. 
Os jovens buscam no ambiente da universidade crescimento pessoal e a conquista de uma profissão, no entanto, este meio social pode propiciar o início e a manutenção do consumo excessivo de álcool e outras drogas lícitas e ilícitas ${ }^{(13)}$.

No intuito de minimizar as consequências geradas pelo abuso de álcool e tabaco, se faz necessária a efetivação na prática de políticas públicas já existentes, pois são meios importantes para reduzir as desigualdades sociais e econômicas, de modo a assegurar o acesso equitativo a bens e serviços, inclusive a atenção à saúde ${ }^{(14)}$.

Neste contexto, enfatiza-se a necessidade de ações de promoção da saúde, compreendidas como estratégias de articulação transversal com enfoque nos determinantes do processo saúde-doença da população e nas diferenças entre necessidades, territórios e culturas presentes no Brasil, a fim de construir mecanismos que diminuam as situações de vulnerabilidade, e insiram a participação e o controle sociais na gestão das políticas públicas $^{(15)}$.

Desta forma, este estudo tem relevância ao tentar investigar e compreender as características e o perfil dos acadêmicos do curso de Enfermagem, preocupação que ainda não recebeu atenção de estudiosos. A partir da pesquisa, pretende-se chamar a atenção para futuras intervenções no combate ao tabagismo e ao álcool.

Assim, o objetivo deste estudo foi estimar a prevalência e fatores associados ao consumo de álcool e tabaco entre estudantes do curso de Enfermagem do Centro Universitário do Distrito Federal - UDF.

\section{MÉTODOS}

Trata-se de um estudo epidemiológico de corte transversal, realizado aleatoriamente com estudantes de ambos os sexos do curso de Enfermagem do Centro Universitário do Distrito Federal - UDF. Foram convidados a participar $100 \%$ dos acadêmicos matriculados, totalizando uma mostra de 974 participantes de um universo de 1.883 acadêmicos regularmente matriculados entre o $1^{\circ} \mathrm{e} 8^{\circ}$ semestre.

Para participar do estudo os alunos deveriam estar matriculados no curso de Enfermagem e estarem presentes na sala de aula no momento da coleta dos dados, onde os pesquisadores foram uma vez para realizar a coleta.

A coleta de dados foi realizada no período de agosto a outubro de 2016 por pesquisadores treinados, por meio da aplicação de um questionário estruturado de autopreenchimento e sem identificação do acadêmico, com perguntas referentes aos hábitos de vida relacionados à saúde. As variáveis: idade, sexo, tipo de escola no ensino médio (escola pública, escola particular, parcialmente em escola particular ou outras - escolas comunitárias), nível socioeconômico (ABEP - Associação Brasileira de Empresas de Pesquisa - 2014 - http://www.abep.org/) $)^{(16)}$, local de moradia (com pais ou parentes, pensionato, casa/apartamento dividido com amigos, sozinho ou outros), frequência de uso do álcool (nunca bebeu, mensal ou menos, 2-4 vezes por mês, 2-3 vezes por semana, e quatro ou mais vezes por semana) e tabaco (nunca fumou, experimentou, fuma em festa ou fim de semana, já foi fumante, e fumante atual), quando adquiriu o hábito de beber e de fumar (antes ou depois de ingressar na instituição) e autopercepção de saúde (excelente, muito boa, boa, regular e ruim). O questionário foi aplicado no intervalo das aulas, numa sala com capacidade para 60 alunos, de maneira que pudesse acomodar os alunos de cada turma com distância entre si capaz de preservar a privacidade das respostas, assim, após o seu 
preenchimento o mesmo foi colocado dentro de um envelope pardo apenas com identificação das turmas e o turno (matutino, vespertino e noturno).

Na sala de aula do curso de Enfermagem, antes de o questionário ser entregue junto com o Termo de Consentimento Livre e Esclarecido (TCLE), os pesquisadores se apresentaram ao docente responsável pela turma, a quem previamente foi explicado os objetivos e a metodologia da pesquisa. Posteriormente, os pesquisadores se apresentaram à turma e explicaram os objetivos, a metodologia, a importância do estudo e fizeram o convite para participarem da pesquisa.

Para a avaliação do consumo de álcool utilizou-se uma pergunta sobre a frequência em que o estudante bebia. Para estudantes que relataram algum tipo de consumo foi utilizado o questionário CAGE $^{(17)}$, um instrumento de ampla utilização que classifica casos suspeitos de alcoolismo por meio de respostas afirmativas a duas ou mais de suas perguntas. O mesmo é composto por quatro questões representadas pelas palavras-chave associadas a cada letra: C - Cut Down (diminuir ingestão); A - Annoyed (irritado); G - Guilty (culpado); E - EyeOpener (necessidade de beber ao acordar para evitar a ressaca). Quando se obtém duas ou mais afirmações, ocorre a confirmação da suspeita de uso abusivo de álcool. A validação do teste CAGE no Brasil encontrou uma sensibilidade de $88 \%$ e uma especificidade de $83 \%{ }^{(18)}$.

Posteriormente, o consumo de álcool foi operacionalizado em dois grupos, sendo considerados "consumidores" aqueles indivíduos que relataram beber pelo menos uma vez ao mês e o outro grupo, "não consumidores", ou seja, aqueles indivíduos que nunca utilizam álcool.

Em relação ao tabagismo, para aqueles que relataram "sim", foi questionado se eles adquiriram o hábito de fumar antes ou depois do ingresso na Universidade, assim, a análise dividiu-se em dois grupos: os fumantes, ou seja, aqueles que relataram consumir cigarro em festas ou fins-de-semana, e aqueles que fumam regularmente. Por outro lado, consideramos como não fumantes aqueles que não consomem cigarro, mesmo que já tenham sido fumantes.

Os dados foram duplamente digitados e checados em relação a possíveis inconsistências. As análises estatísticas foram realizadas no pacote estatístico Stata 9.0. De início foram realizadas as análises descritivas (com médias e proporções) e, por fim, análises de associação através dos testes de qui-quadrado para heterogeneidade e regressões logísticas, com nível de significância de 0,05 e intervalo de confiança de 95\%.

Este estudo seguiu as diretrizes da Resolução n 466/2012 do Conselho Nacional de Saúde e foi aprovado pelo Comitê de Ética em Pesquisa do Centro Universitário do Distrito Federal - UDF, recebendo aprovação (CAAE: 59713316.0.0000.5650) no ano de 2016.

\section{RESULTADOS}

Do total de 1.883 acadêmicos matriculados, participaram do estudo 974 estudantes, sendo, $78,8 \%$ do sexo feminino e $21,2 \%$ do sexo masculino. A média de idade dos alunos foi de $25,4 \pm 6,1$ anos, sendo que $62,6 \%$ tinham entre 20 e 29 anos. A Tabela 1 descreve a amostra segundo as características demográficas, socioeconômicas e de saúde. Em relação ao tipo de escola no ensino médio, 71,2\% estudaram em escola pública e 68,5\% moram com pais ou parentes. Em relação à autopercepção de saúde, $43,4 \%$ dos participantes consideram sua saúde como "boa" e 25,4\% como "muito boa". 
Tabela 1: Descrição da amostra segundo variáveis demográficas, socioeconômicas e percepção de saúde. Brasília, DF, Brasil, 2016.

\begin{tabular}{|c|c|c|}
\hline Variáveis & $\mathbf{N}$ & $\%$ \\
\hline \multicolumn{3}{|l|}{ Sexo } \\
\hline Feminino & 767 & 78,8 \\
\hline Masculino & 207 & 21,2 \\
\hline \multicolumn{3}{|l|}{ Idade } \\
\hline$<20$ & 152 & 15,6 \\
\hline 20 a 29 & 610 & 62,6 \\
\hline$>30$ & 212 & 21,8 \\
\hline \multicolumn{3}{|l|}{ Tipo de escola no ensino médio } \\
\hline Escola Pública & 693 & 71,2 \\
\hline Escola Particular & 200 & 20,5 \\
\hline Parcialmente em escola particular & 43 & 4,4 \\
\hline Outra & 38 & 3,9 \\
\hline \multicolumn{3}{|l|}{ Nível socioeconômico } \\
\hline A & 41 & 4,2 \\
\hline B & 269 & 27,6 \\
\hline C & 552 & 56,7 \\
\hline D & 112 & 11,5 \\
\hline \multicolumn{3}{|l|}{ Local de moradia } \\
\hline Pais ou parentes & 667 & 68,5 \\
\hline Pensionato & 3 & 0,4 \\
\hline Casa dividida com amigos & 55 & 5,7 \\
\hline Sozinho & 82 & 8,4 \\
\hline Outro & 167 & 17,2 \\
\hline \multicolumn{3}{|l|}{ Autopercepção de saúde } \\
\hline Excelente & 109 & 11,2 \\
\hline Muito boa & 247 & 25,4 \\
\hline Boa & 423 & 43,4 \\
\hline Regular & 174 & 17,8 \\
\hline Ruim & 21 & 2,2 \\
\hline Total & 974 & 100 \\
\hline
\end{tabular}

O consumo de álcool e o tabaco estão descritos na Tabela 2. O consumo de álcool, pelo menos uma vez ao mês, foi relatado por $37,2 \%$ da amostra, não mostrando diferença segundo o sexo.

Tabela 2: Consumo de álcool e tabaco entre estudantes, segundo o sexo, do curso de Enfermagem do Centro Universitário do Distrito Federal. Brasília, DF, Brasil, 2016.

\begin{tabular}{|c|c|c|c|c|c|c|}
\hline \multirow{2}{*}{ Variáveis } & \multicolumn{2}{|c|}{ Total } & \multicolumn{2}{|c|}{ Feminino } & \multicolumn{2}{|c|}{ Masculino } \\
\hline & $\mathbf{N}$ & $\%$ & $\mathbf{N}$ & $\%$ & $\mathbf{N}$ & $\%$ \\
\hline Consumo de álcool & & & \multicolumn{4}{|c|}{$P=0,652$} \\
\hline Nunca bebeu & 340 & 34,9 & 265 & 34,6 & 75 & 36,2 \\
\hline Mensal ou menos & 363 & 37,2 & 292 & 38,1 & 71 & 36,2 \\
\hline $2-4$ vezes por mês & 200 & 20,5 & 153 & 20,0 & 47 & 22,7 \\
\hline $2-3$ vezes por semana & 62 & 6,4 & 50 & 6,5 & 12 & 5,8 \\
\hline 4 ou mais vezes por semana & 9 & 0,9 & 7 & 0,9 & 2 & 1,0 \\
\hline Total & 974 & 100 & 767 & 100 & 207 & 100 \\
\hline Consumo de tabaco & & & \multicolumn{2}{|c|}{$P=0,144$} & & \\
\hline Nunca fumou & 751 & 77,1 & 598 & 77,9 & 153 & 73,9 \\
\hline Experimentou, mas não regular & 116 & 11,9 & 86 & 11,2 & 30 & 14,4 \\
\hline Fuma em festa ou final de semana & 50 & 5,1 & 38 & 4,9 & 12 & 5,8 \\
\hline Já foi fumante, mas parou & 32 & 3,2 & 26 & 3,3 & 6 & 2,9 \\
\hline Fumante atual & 25 & 2,5 & 19 & 2,4 & 6 & 2,9 \\
\hline Total & 974 & 100 & 767 & 100 & 207 & 100 \\
\hline
\end{tabular}


Em relação ao tabaco, 5,8\% dos estudantes homens relataram que fumam em festas ou final de semana. Este percentual foi menor no sexo feminino (4,9\%). Entre os acadêmicos entrevistados, 3,2\% disseram que já foram fumantes, mas pararam com o consumo, e aproximadamente $77,1 \%$ da amostra relataram nunca ter fumado.

A Figura 1 demonstra a suspeita para o alcoolismo de acordo com o teste CAGE, onde 3,0\% dos alunos apresentaram resultado positivo, sendo 3,9\% entre os homens e $2,7 \%$ entre as mulheres.

Figura 1: Prevalência dos indicadores de problemas com álcool segundo sexo. Brasília, DF, Brasil, 2016.

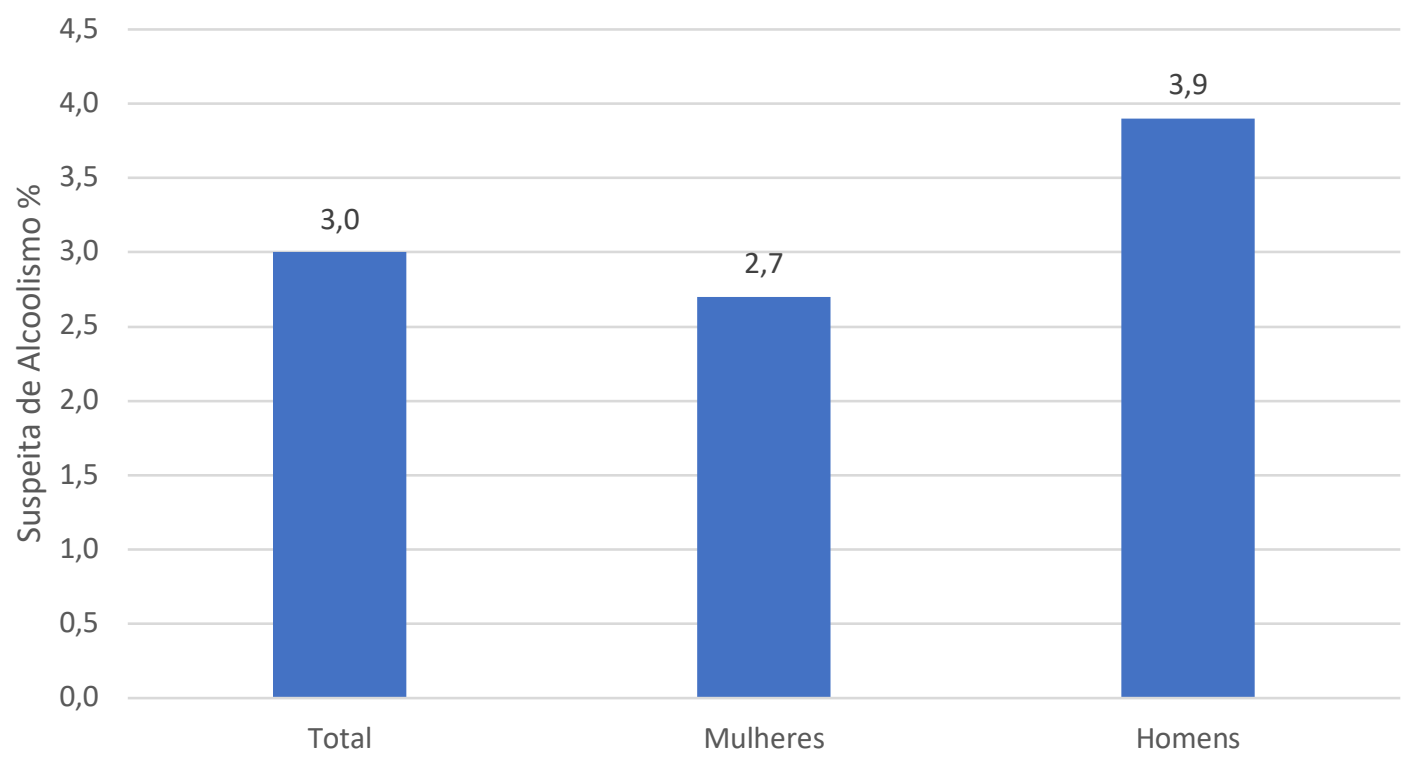

Ainda entre aqueles acadêmicos que ingeriram álcool, as mulheres $(94,2 \%)$ e homens $(91,7 \%)$ relataram que este hábito foi adquirido antes de ingressar na universidade, não mostrando diferença significativa segundo o sexo. Tal quadro repetiu-se quando analisado o tabagismo. Notamos que $81,8 \%$ das mulheres e $68,8 \%$ dos homens adquiriram o hábito de fumar antes de ingressar na universidade $(p<0,05)$ (Tabela 3$)$.

Tabela 3: Descrição da amostra segundo o momento que iniciou a consumir álcool e/ou cigarro. Brasília, DF, Brasil, 2016.

\begin{tabular}{ccc}
\hline \multicolumn{1}{c}{ Variáveis } & Feminino N (\%) & Masculino N (\%) \\
\hline Quando adquiriu o hábito de beber & & $\mathrm{P}=0,282$ \\
Antes de ingressar na Universidade & $473(94,2)$ & $121(91,7)$ \\
Depois de ingressar na Universidade & $29(5,8)$ & $11(8,3)$ \\
\hline Total & $\mathbf{5 0 2 ( 1 0 0 , 0 )}$ & $\mathbf{1 3 2 ( 1 0 0 , 0 )}$ \\
\hline Quando adquiriu o hábito de fumar & $117(81,8)^{*}$ & $33(68,8)$ \\
Antes de ingressar na Universidade & $26(18,2)$ & $15(31,3)$ \\
Depois de ingressar na Universidade & $\mathbf{1 4 3 ( 1 0 0 , 0 )}$ & $\mathbf{4 8 ( 1 0 0 , 0 )}$ \\
\hline Total & & 0,056 \\
\hline
\end{tabular}

* Teste Qui quadrado de heterogeneidade.

A Tabela 4 mostra a associação entre o consumo de álcool e o tabagismo com variáveis demográficas, socioeconômicas e de saúde. Os acadêmicos na faixa etária dos 20 a 29 anos apresentaram uma prevalência maior para o consumo de álcool $(67,5 \%)$ e para o tabagismo (21,3\%). Acadêmicos que fizeram o ensino médio parcialmente em escolas particulares apresentaram maior chance de consumo de álcool e tabaco. Da mesma forma, o grupo de nível socioeconômico B também apresentou maior probalidade de consumir álcool e tabaco. 
Tabela 4: Associação do consumo de álcool e tabagismo com variáveis demográficas, socioeconômicas e de saúde. Brasília, DF, Brasil, 2016.

\begin{tabular}{|c|c|c|c|c|c|c|}
\hline \multirow{2}{*}{ Variável } & \multicolumn{2}{|c|}{ Consumo de álcool } & \multirow[t]{2}{*}{ p-valor } & \multicolumn{2}{|c|}{ Tabagismo } & \multirow{2}{*}{ p-valo } \\
\hline & $\%$ & OR (IC95\%) & & $\%$ & OR (IC95\%) & \\
\hline \multicolumn{7}{|l|}{ Sexo } \\
\hline Feminino & 65,5 & 1,00 & \multirow{2}{*}{0,653} & 18,6 & 1,00 & \multirow{2}{*}{0,150} \\
\hline Masculino & 63,8 & $0,93(0,77-1,28)$ & & 23,2 & $1,32(0,91-1,91)$ & \\
\hline \multicolumn{7}{|l|}{ Idade } \\
\hline$<20$ & 57,2 & 1,00 & \multirow{3}{*}{0,054} & 21,7 & 1,00 & \multirow{3}{*}{0,299} \\
\hline 20 a 29 & 67,5 & $1,55(1,08-2,24)$ & & 21,3 & $0,92(0,60-1,42)$ & \\
\hline$>30$ & 63,7 & $1,31(0,86-2,01)$ & & 16,0 & $0,69(0,40-1,17)$ & \\
\hline \multicolumn{7}{|l|}{ Tipo de escola no ensino médio } \\
\hline Escola Particular & 71,0 & 1,00 & \multirow{4}{*}{0,158} & 22,5 & 1,00 & \multirow{4}{*}{0,097} \\
\hline Escola Pública & 62,9 & $0,69(0,49-0,98)$ & & 18,2 & $0,77(0,52-1,12)$ & \\
\hline Parcialmente em escola particular & 69,8 & $0,94(0,46-1,93)$ & & 32,6 & $1,67(0,81-3,41)$ & \\
\hline Outra & 68,4 & $0,89(0,42-1,87)$ & & 15,8 & $0,65(0,25-1,64)$ & \\
\hline \multicolumn{7}{|l|}{ Nível socioeconômico } \\
\hline A & 68,3 & 1,00 & \multirow{4}{*}{0,940} & 34,2 & 1,00 & \multirow{4}{*}{0,072} \\
\hline B & 65,8 & $0,89(0,44-1,80)$ & & 20,1 & $0,48(0,24-0,99)$ & \\
\hline C & 64,9 & $0,86(0,43-1,69)$ & & 19,4 & $0,46(0,24-0,91)$ & \\
\hline D & 63,4 & $0,80(0,78-1,70)$ & & 14,3 & $0,32(0,14-0,74)$ & \\
\hline \multicolumn{7}{|l|}{ Local de moradia } \\
\hline Casa dividida com amigos & 72,7 & 1,00 & \multirow{4}{*}{0,224} & 16,4 & 1,00 & \multirow{4}{*}{0,02} \\
\hline Pais ou parentes & 66,3 & $0,74(0,40-1,36)$ & & 22,8 & $1,50(0,72-3,15)$ & \\
\hline Sozinho & 59,8 & $0,56(0,27-1,17)$ & & 11,0 & $0,73(0,23-1,70)$ & \\
\hline Outro & 60,6 & $0,58(0,30-1,13)$ & & 12,4 & $0,72(0,31-1,68)$ & \\
\hline \multicolumn{7}{|l|}{ Autopercepção de saúde } \\
\hline Boa & 68,8 & 1,00 & \multirow{5}{*}{0,051} & 18,9 & 1,00 & \multirow{5}{*}{0,791} \\
\hline Excelente & 56,9 & $0,60(0,40-0,92)$ & & 21,1 & $1,15(0,68-1,93)$ & \\
\hline Muito Boa & 66,4 & $0,90(0,64-1,25)$ & & $21,91,20(0,81-1,77)$ & & \\
\hline Regular & 58,6 & $0,64(0,45-0,93)$ & & $17,20,89(0,56-1,41)$ & & \\
\hline Ruim & 71,4 & $1,13(0,43-2,98)$ & & $19,11,01(0,33-3,07)$ & & \\
\hline
\end{tabular}

Os estudantes que moram com os pais ou parentes apresentaram maior prevalência de tabagismo $(22,8 \%)$ e de consumo de álcool $(66,3 \%)$ e, em contraste, quanto menor a autopercepção de saúde maior foi a probabilidade de consumo de álcool.

\section{DISCUSSÃO}

O presente estudo é um dos primeiros a respeito do tabagismo e do consumo de álcool em uma amostra representativa de estudantes universitários na cidade de Brasília-DF. Os resultados aqui apresentados podem servir de subsídios para futuras intervenções que objetivem estimular hábitos saudáveis entre os estudantes.

Um trabalho realizado em mais de 100 cidades brasileiras com mais de 200 mil habitantes mostrou que o consumo de álcool é muito relevante na faixa etária de 18 a 24 anos e que 15,5\% dessa população referem dependência ${ }^{(19)}$. Entre estudantes de Medicina, esse percentual variou $25 \%$ de consumo exagerado até $98 \%$ em algumas regiões, dependendo da amostra e da forma de coleta dos dados ${ }^{(19)}$. Estudantes de Juiz de Fora, por exemplo, apresentaram prevalência de $25 \%$ de consumo exagerado ${ }^{(20)}$.

A juventude brasileira tem cultivado o hábito de se embriagar. No Brasil, estudos epidemiológicos mostram que o consumo de álcool é maior entre universitários do que entre estudantes do ensino médio(20). Em nosso 
estudo, observamos que a maioria dos estudantes iniciaram o consumo de álcool e/ou tabaco antes de ingressar na universidade, o que podemos associar com a influência do grupo de amigos e famílias.

Esses dados do nosso estudo são relevantes para mostrar que medidas preventivas devem ser iniciadas já na escola, onde o uso precoce do álcool tem sido motivo de preocupação nas escolas e ambiente familiar, onde o maior desafio está na criação de novas políticas públicas para a prevenção desse problema.

A educação começa em casa, e os pais não podem pensar que é dever da escola educar. O papel da escola é ensinar. Verbos diferentes, ações diferentes. Acreditamos que investimentos na adolescência como projetos focando a temática no ensino médio só terá efeito com a participação e intervenção dos pais em conjunto com a escola. Cabe à escola inserir o adolescente e sua família nos projetos sociais que visem o combate ao uso precoce de drogas, aceitando também a ajuda da comunidade.

A escola tem o dever de cumprir seu papel social em conjunto com os pais dos adolescentes, onde palestras feitas por "ex-dependentes" podem servir de demonstração dos graves efeitos mediatos e imediatos do álcool.

A Sociedade de Pediatria de São Paulo lançou a campanha gravidez sem álcool, com o objetivo de alertar sobre a Síndrome Alcoólica Fetal (SAF), no intuito de evitar que bebês nasçam com deficiência por causa de bebidas alcoólicas.

Outros ambientes, além da escola, também podem servir como cenário para o emprego de ações de prevenção de drogas, tais como a comunidade e o serviço de atenção à saúde, através de médicos pediatras e Centro de Apoio e Atendimento ao Adolescente.

Estima-se que o uso de álcool esteja aumentando em decorrência do estilo de vida, ansiedade, estresse, depressão e baixa autoestima ${ }^{(20)}$. Os universitários estão mais expostos aos ambientes em que o álcool é mais fácil. Isso aponta uma necessidade maior de o ambiente acadêmico oferecer apoio e treinamento efetivo para os estudantes pararem de beber ${ }^{(19)}$.

Um estudo realizado com 174 alunos de uma universidade pública de São Luís-MA observou que 59\% dos participantes responderam já ter consumido de forma abusiva algum tipo de bebida alcoólica, dos quais 20,7\% relataram já ter se embriagado nos últimos 19 dias, e 14,9\%, nos últimos cinco dias que antecedem a coleta ${ }^{(21)}$.

Em contrapartida, um estudo realizado na cidade de Piracicaba, interior do estado de São Paulo, avaliou o consumo do álcool e a qualidade de vida em universitários, sendo sua amostra composta por 170 indivíduos de ambos os sexos, onde observou-se que $68,20 \%$ dos participantes faziam uso de baixo risco do álcool, $21,80 \%$ faziam uso de alto risco, e 5,29\% dos alunos se encontravam em provável dependência do álcool ${ }^{(16)}$.

Do mesmo modo, resultados encontrados por uma pesquisa realizada em uma universidade pública em São Paulo com 112 alunos do curso de Enfermagem, com idades entre 17 e 28 anos, verificaram que 79,5\% dos indivíduos se encontravam em baixo risco de dependência do álcool(17).

Com relação ao grupo amostral, houve um predomínio do sexo feminino. Em concordância com os resultados do presente estudo, um estudo anterior ${ }^{(18)}$ que investigou o consumo de álcool verificou que a maioria era do sexo feminino $(61,6 \%)$ e apenas $38,4 \%$ da amostra era do sexo masculino, assim como em vários outros trabalhos sobre o uso do álcool, o que corrobora com os achados ${ }^{(19-20)}$.

Um estudo realizado com acadêmicos do curso de Enfermagem em uma instituição privada da cidade de Montes Claros-MG mostrou um consumo de álcool de 77,4\%, com prevalência discretamente superior entre 
indivíduos do nível socioeconômico $\mathrm{B}^{(21)}$. Em nosso estudo foi observado que o grupo de nível socioeconômico $\mathrm{C}$ apresentou maior probalidade de consumir álcool e tabaco.

Uma explicação para o alto padrão de consumo de álcool por mulheres é que com a independência financeira feminina, a conquista de determinados direitos e postos na sociedade e a luta por igualdade entre os gêneros fez com que a mulher adotasse alguns hábitos tipicamente masculinos dentre ele o consumo de álcool(21).

Em uma pesquisa realizada no Chile com 490 universitários ${ }^{(22)}$ e na Colômbia ${ }^{(23)}$ com 1.324 universitários, revela que os homens dos cursos das áreas de ciências sociais consomem mais tabaco do que as mulheres.

O tabagismo foi observado em $17 \%$ dos estudantes de medicina de Vassouras ${ }^{(24)}$, número bem superior aos demais levantamentos semelhantes realizados com estudantes universitários em Pouso Alegre-MG que mostraram prevalência de $7,8 \%(25)$.

Notamos em nosso estudo que indivíduos apresentaram associação entre consumo de álcool e percepção de saúde classificada como "ruim", esse fato demonstra que essa percepção negativa do estado de saúde exerce influência na decisão do indivíduo de procurar um médico ou um serviço de saúde ${ }^{(24)}$, e que essa percepção negativa de saúde aumenta a prevalência de comportamentos de risco.

A autoavaliação de saúde, segundo alguns autores, é considerada um importante fator na predição de problemas de saúde que só seriam detectados mais tardiamente ${ }^{(24)}$. A autoavaliação do estado de saúde é um importante indicador do constructo multidimensional da saúde, é portanto um indicador subjetivo e engloba tanto componentes físicos quanto emocionais dos indivíduos, além de aspectos do bem-estar e da satisfação com a própria vida.

Propagandas de bebidas alcoólicas influenciam o uso dessas substâncias pelos jovens, uma vez que elas, na maioria das vezes, são voltadas para esse público. No Brasil, quase não há programas de alerta aos perigos do abuso do álcool, e as propagandas sobre bebidas alcoólicas quase não possuem restrições ao serem veiculadas ao público ${ }^{(19)}$.

A limitação desta pesquisa diz respeito ao desenho transversal do estudo que impossibilitou a identificação da precedência temporal dos fatores estudados, comprometendo as evidências de relações de causa e efeito. Assim, considera-se importante a ampliação de estudos sobre os malefícios causados pelo uso abusivo do álcool e tabaco entre universitários da área da saúde, sendo estes detentores do conhecimento e promotores da saúde.

Estudos futuros devem investigar outros comportamentos relacionados à saúde e levantar informações sobre a presença ou não de doenças, uma vez que esta variável não foi investigada neste estudo. Assim, sugeremse propostas como o esclarecimento das consequências do consumo de álcool e tabaco, o envolvimento da família como formadora da consciência e da conduta do jovem por meio do fortalecimento dos vínculos familiares, a inclusão da escola na discussão dos efeitos dessas drogas e a continuidade das estratégias de educação em saúde voltadas para o consumo do álcool e tabaco.

\section{CONCLUSÃO}

A prevalência do consumo de álcool entre os estudantes de Enfermagem é elevada, e a sua frequência é maior entre as mulheres $(65,5 \%)$. Os dados sugerem a necessidade de intervenções no meio acadêmico. 
Entretanto, cabe destacar que ações para a população adolescente como um todo devem ser priorizadas, pois mais de $80 \%$ dos alunos adquiriram os hábitos antes de ingressar na universidade.

\section{REFERÊNCIAS}

1. United Nations Office on Drugs and Crime. World Drug Report 2016 [Internet]. Viena (SW): UNODC; 2016 [cited 2018 Dec 31 ]. Available from: https://www.unodc.org/wdr2016/.

2. Nascimento PF, Junior GA. Implicações do uso de drogas e a condição de saúde dos caminhoneiros. Psicologia e Saúde em Debate. 2016;2:104-116.

3. Medeiros LKA. As marcas dos hospitais Psiquiátricos: um relato de experiência. Psicologia e Saúde em Debate. 2015;1(2):20-34. 4. Wright MGM, Cumsille F, Padilha MI, Ventura CA, Sapag J, Brands B, et al. International research capacity building program for health related professionals to study the drug phenomenon in Latin America and the Caribbean. Texto Contexto Enferm. 2015;24:17-25.

5. Arora A, Kannan S, Gowri S, Choudhary S, Sudarasanan S, Khosla PP. Substance abuse amongst the medical graduate students in a developing country. Indian J Med Res. 2016;143(1):101-3.

6. Arria AM, Caldeira KM, Allen HK, Vincent KB, Bugbee BA, O'Grady KE. Drinking like an adult? Trajectories of alcohol use patterns before and after college graduation. Alcohol Clin Exp Res. 2016;40(3):583-90.

7. World Health Organization. Global reporto on trends in prevalence of tobacco smoking. Geneva: WHO, 2015.

8. Pinto MT, Pichon-Riviere A, Bardach A. The burden of smoking-related diseases in Brazil: mortality, morbidity and costs. Cad Saúde Pública. 2015;31(6):1283-1297.

9. Brasil, Secretaria Nacional de Políticas sobre Drogas. I Levantamento nacional sobre o uso de álcool, tabaco e outras drogas entre universitários das 27 capitais brasileiras. Brasília, DF, 2010.

10. Santos MVF, Pereira DS, Siqueira MM. Uso de álcool e tabaco entre estudantes de psicologia da Universidade Federal do Espírito Santo. Jornal Bras Psiq. 2013;62(1):22-30

11. Haas AL, Smith SK, Kagan K, Jacob T. Pre-college pregaming: practices, risk factors, and relationship to other indices of problematic drinking during the transition from high school to college. Psychology of Addictive Behaviors. 2012;26(4):931-938.

12. McBride NM, Barrett B, Moore KA, Schonfeld L. The role of positive alcohol expectancies in underage binge drinking among college students. J Am Coll Health. 2014;62(6):370-9

13. Bottorff JL, Haines-Saah R, Kelly MT, Oliffe JL, Torchalla I, Poole N, et al. Gender, smoking and tobacco reduction and cessation: a scoping review. Int J Equity Health. 2014;13:114

14. Chiauzzi E, Dasmahapatra P, Black RA. Risk behaviors and drug use: a latente class analysis of heavy episodic drinking in firt-year college students. Psychology of Addictive Behaviors. 2013;27(4):974-85

15. Ministério da Saúde. Política Nacional de Promoção da Saúde (3ạ ed.). Brasília, DF, 2010.

16. Associação Brasileira de Empresas de Pesquisa. Critério de classificação econômica Brasil, 2016.

17. Ramis TR, Mielke GI, Habeyche EC, Oliz MM, Azevedo MR, Hallal PC. Tabagismo e consumo de álcool em estudantes universitários: prevalência e fatores associados. Rev bras epidemiol. 2012;15(2):376-385.

18. Barbosa FL, Barbosa RL, Aguiar DL, Ribeiro AC, Figueiredo IA, et al. Uso de álcool entre estudantes de Medicina da Universidade Federal do Maranhão. Rev. Bras. Educ. Med. 2016;37(1):89-95.

19. Martinho AF, Tonin CL, Nunes LM, Novo NF, Hubner CVK. Uso de álcool e drogas por acadêmicos dos cursos de enfermagem, biologia e medicina na pontifica universidade católica de São Paulo. Rev. Fac. Ciênc. Méd. Sorocaba. 2016;11(1):11-15.

20. Pinheiro MA, Torres LF, Bezerra M, Cavalcante RC, Alencar RD. Prevalência e fatores associados ao consumo de álcool e tabaco entre estudantes de medicina no nordeste do Brasil. Rev.Bras. Educ. Méd. 2017;41(2):231-250.

21. Funai A, Pillon SC. Uso de bebidas alcoólicas e aspectos religiosos em estudantes de enfermagem. Rev Eletrônica Enferm. 2014;13(1):24-9.

22. Grazia J, Faivovich D, Fálcon F, Díaz R, Yentzen G. Prevalencia de tabaquismo y actitud de cambio frente al hábito tabáquico en universitarios chilenos: Importancia de la formación médica. Rev Chil Salud Pública. 2012;13(2):72-81.

23. Tafúr LA, Ordoñez G, Millán JC, Varela JM, Rebellón P. Prevalencia de tabaquismo en estudiantes recién ingressados a la Universidad Santiago de Cali. Colomb Med. 2013; 37:126-132.

24.Werneck FA, Souza NE, Cartier LCM, Lourenço C, Delgado PNM, Menezes C. Prevalência do tabagismo entre os estudantes de Medicina da Universidade Severino Sombra. Rev. Saúde.2016;07(2):08-11.

25. Almeida A, Beraldo CL, Magalhães EF, Lima JPR, Guimarães ML. Tabagismo e sua relação com dados sociais uso de álcool, café e prática de esportes, em estudantes da Universidade do Vale do Sapucaí (UNIVÁS), Pouso Alegre, MG. Rev. Med. Minas Gerais. 2016;21(2):168-173. 